\title{
Knockdown of FOXA2 enhances the osteogenic differentiation of bone marrow- derived mesenchymal stem cells partly via activation of the ERK signalling pathway
}

\author{
Chenyi Ye ${ }^{1,2}$, Mo Chen ${ }^{3}$, Erman Chen ${ }^{1,2}$, Weixu Li ${ }^{1,2}$, Shengdong Wang $\mathbb{1}^{1,2}$, Qianhai Ding ${ }^{1,2}$, Cong Wang ${ }^{1,2}$, \\ Chenhe Zhou ${ }^{1,2}$, Lan Tang ${ }^{1,2}$, Weiduo Hou ${ }^{1,2}$, Kai Hang ${ }^{1,2}$, Rongxin He ${ }^{1,2}$, Zhijun Pan ${ }^{1,2}$ and Wei Zhang ${ }^{1,2}$
}

\begin{abstract}
Forkhead box protein A2 (FOXA2) is a core transcription factor that controls cell differentiation and may have an important role in bone metabolism. However, the role of FOXA2 during osteogenic differentiation of bone marrowderived mesenchymal stem cells (BMSCs) remains largely unknown. In this study, decreased expression of FOXA2 was observed during osteogenic differentiation of rat BMSCs (rBMSCs). FOXA2 knockdown significantly increased osteoblast-specific gene expression, the number of mineral deposits and alkaline phosphatase activity, whereas FOXA2 overexpression inhibited osteogenesis-specific activities. Moreover, extracellular signal-regulated protein kinase (ERK) signalling was upregulated following knockdown of FOXA2. The enhanced osteogenesis due to FOXA2 knockdown was partially rescued by an ERK inhibitor. Using a rat tibial defect model, a rBMSC sheet containing knocked down FOXA2 significantly improved bone healing. Collectively, these findings indicated that FOXA2 had an essential role in osteogenic differentiation of BMSCs, partly by activation of the ERK signalling pathway.
\end{abstract}

\section{Introduction}

Fracture delayed union or non-union is one of the most common complications following fixation, which continues to be a main challenge for orthopaedic surgeons. It has been reported that the overall percentage of delayed union or non-union can be as high as $4.4 \%$ of all open fractures ${ }^{1}$. Despite significant advances in treatments including autologous bone grafting, artificial bone grafting and commercially available recombinant human bone morphogenetic protein-2, the prognoses for delayed

\footnotetext{
Correspondence: Rongxin He (herongxin@zju.edu.cn) or Zhijun Pan (zrpzj@zju. edu.cn) or Wei Zhang (zhangweilook@zju.edu.cn)

'Department of Orthopedic Surgery, The Second Affiliated Hospital, School of Medicine, Zhejiang University, No. 88, Jiefang Road, Hangzhou 310009, China ${ }^{2}$ Orthopedics Research Institute of Zhejiang University, No. 88, Jiefang Road, Hangzhou 310009, China

Full list of author information is available at the end of the article. These authors contributed equally: Chenyi Ye, Mo Chen and Erman Chen Edited by Y. Shi
}

union or non-union are still not optimal ${ }^{2-5}$. Thus, there remains an urgent need to develop new and effective methods to accelerate bone healing.

As a major contributor to bone formation, mesenchymal stem cells (MSCs) have been reported to have a key role during fracture healing and have emerged as the most promising candidate for tissue repair ${ }^{6-8}$. MSCs are regulated by transcription factors and possess self-renewal capabilities, together with the potential to differentiate into a variety of bone formation-related cell types such as osteoblasts and chondrocytes ${ }^{9-11}$. During the fracturehealing process, MSCs are recruited to the fracture area and initiate proliferation and differentiation under the stimulation of the local microenvironment ${ }^{9,10,12}$. The underlying mechanism of osteogenesis, however, remains largely unknown. A better understanding of the osteogenic differentiation of MSCs, especially the involvement

\section{(c) The Author(s) 2018}

(c) (i) Open Access This article is licensed under a Creative Commons Attribution 4.0 International License, which permits use, sharing, adaptation, distribution and reproduction cc. in any medium or format, as long as you give appropriate credit to the original author(s) and the source, provide a link to the Creative Commons license, and indicate if changes were made. The images or other third party material in this article are included in the article's Creative Commons license, unless indicated otherwise in a credit line to the material. If material is not included in the article's Creative Commons license and your intended use is not permitted by statutory regulation or exceeds the permitted use, you will need to obtain permission directly from the copyright holder. To view a copy of this license, visit http://creativecommons.org/licenses/by/4.0/. 
of genetic factors, is necessary for their clinical application.

Forkhead box protein A2 (FOXA2), also known as HNF-3 $\beta$, is a member of the forkhead/winged-helix family of transcription factors (FOXA1, FOXA2 and FOXA3). The members of this family have diverse roles in regulating cell differentiation, development and tissue homoeostasis $^{13,14}$. FOXA2 binds to nucleosomal DNA and is associated with nucleosomal depletion during differentiation ${ }^{15}$. Several studies have reported that FOXA2 closely interacts with several crucial bone metabolismrelated genes including SIRT1, SOX9, SOX17, WNT3 and WNT7b, which suggests a potential role of Foxa2 in osteogenesis $^{16-20}$. Among them, SIRT1 is a repressor of sclerostin and serves as an important regulator of bone mass $^{21}$, whereas WNT3 and WNT7b are required for osteogenesis during biogenesis via $\mathrm{Wnt} / \beta$-catenin signalling $^{22,23}$. Previous studies have also shown an essential role of SOX9 and SOX17 in the proliferation and osteogenesis of stem cells ${ }^{24,25}$. Taken together, there is a potential connection between FOXA2 and bone metabolism, although no related studies have been reported.

In this study, we investigated the effects of FOXA2 on the osteogenic differentiation of bone marrow-derived MSCs (BMSCs). FOXA2 knockdown (KD) enhanced osteogenic differentiation of BMSCs via activation of the extracellular signal-regulated protein kinase (ERK) signalling pathway in vitro, whereas FOXA2 overexpression $(\mathrm{OE})$ inhibited in vitro osteogenic differentiation. Moreover, using a rat tibial osteotomy model, we showed that FOXA2-KD in BMSCs improved bone healing in vivo.

\section{Results}

Level of FOXA2 was decreased in osteogenic-differentiated rBMSCs compared with undifferentiated BMSCs

To determine the expression level of FOXA2 associated with osteogenic differentiation of MSCs, we compared endogenous FOXA2 expression of undifferentiated and osteogenic differentiated rat BMSCs (rBMSCs). Compared with undifferentiated BMSCs, the expression of FOXA2 increased significantly after osteogenic differentiation (Figure 1a-c).

\section{Establishment of FOXA2 OE and KD in rBMSCs}

To clarify the role of FOXA2 during osteogenic differentiation of BMSCs, endogenous FOXA2 was overexpressed in BMSCs or downregulated by lentiviral particles. Based on its effectiveness, shRNA2 was chosen to downregulate the expression of FOXA2 in subsequent experiments. The lentiviral vectors were efficiently used to overexpress or knock down FOXA2 in $>80 \%$ of thirdgeneration rBMSCs, which was quantified by evaluating the ratio of green fluorescent protein (GFP)-positive cells to the total cell number (Fig. 1d). FOXA2 expression was determined by quantitative PCR (qPCR), immunofluorescence (IF), and western blotting 5 days after infection and screening. FOXA2 mRNA and protein levels were significantly up- or downregulated relative to the negative control groups (Figure 1e-i), which was also confirmed by IF analysis (Fig. 1j).

\section{FOXA2 OE and KD did not affect rBMSC proliferation}

To determine whether up- or downregulated FOXA2 expression influenced cell viability and proliferation, rBMSC viability was analysed on days 3, 5, 7 and 14 following infection with Trypan blue and Cell Counting Kit-8 (CCK-8) staining. No significant difference in cell viability or proliferation was detected between the FOXA2 OE and normal control (NC) groups. There was also no significant difference in cell viability or proliferation between the FOXA2-KD and KD-NC groups (Fig. 1k, l).

FOXA2-KD increased the levels of osteospecific genes and proteins, but FOXA2 OE reduced the levels of osteospecific genes and proteins

To assess the role of FOXA2 in osteogenic differentiation, the levels of osteospecific genes including alkaline phosphatase $(A L P)$, osteopontin $(O P N), R U N X 2$, COL1A1 and osteocalcin $(O C N)$ were detected by qPCR. In addition, the protein levels of the osteospecific markers RUNX2 and COL1A1 were evaluated by IF. The results of qPCR revealed that $A L P, O P N, R U N X 2, C O L 1 A 1$, and $O C N$ mRNA levels were significantly higher in the KD group on days 3, 7 and 14 compared with the KD-NC group $(P<0.05)$. Moreover, lower mRNA levels of $A L P$, $O P N, R U N X 2, C O L 1 A 1$ and OCN were detected in the OE group compared with the OE-NC group $(P<0.05)$ (Figure 2a-e). We also used IF to confirm the expression of RUNX2 and COL1A1 proteins and determined that their expressions were increased on day 3 in FOXA2-KD BMSCs (Fig. 2f-h).

FOXA2-KD enhanced calcium deposit formation and ALP activity, whereas FOXA2 OE decreased ALP activity and calcium deposit formation

We evaluated the calcium deposits by Alizarin Red S (ARS) and von Kossa staining. More calcium deposits were present in the KD group than in the KD-NC group on day 10 and less calcium deposits were detected in the OE group than in the OE-NC group (Fig. 2I-K). The activity of ALP, an early marker of osteogenesis, was also examined on day 3 of osteogenic differentiation. Compared with the KD-NC group, increased ALP activity was observed in the KD group $(P<0.05)$, and compared with the OE-NC group, decreased ALP activity was observed in the OE group $(P<0.05)$ (Fig. 2l). 


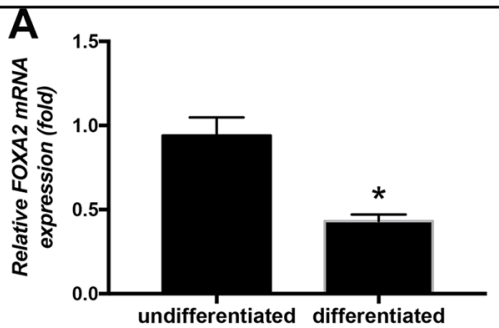

D

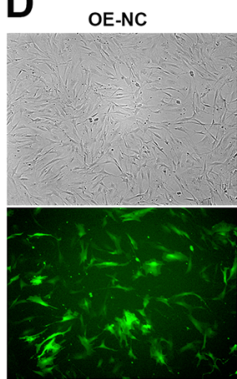

F

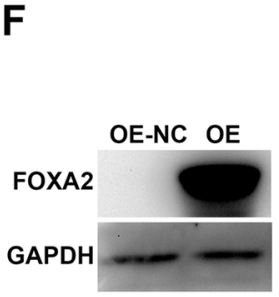

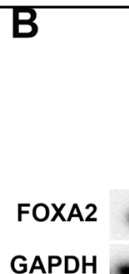

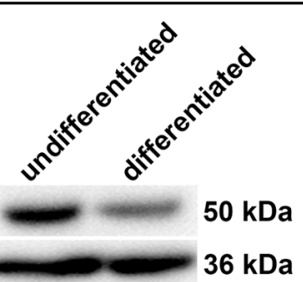

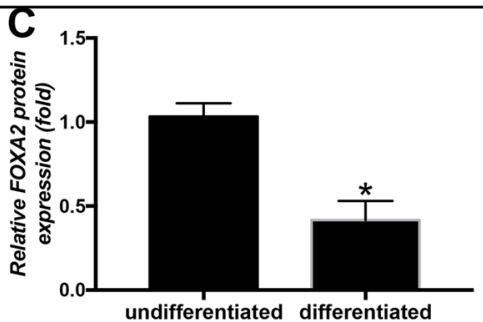

E

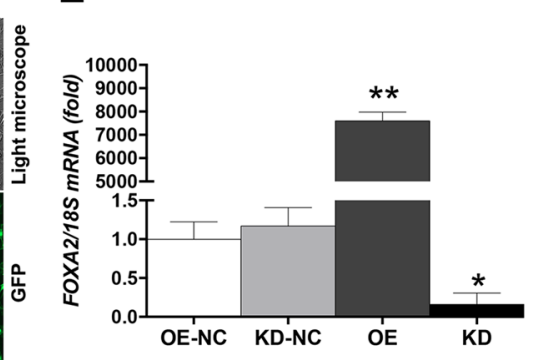

J DAPI

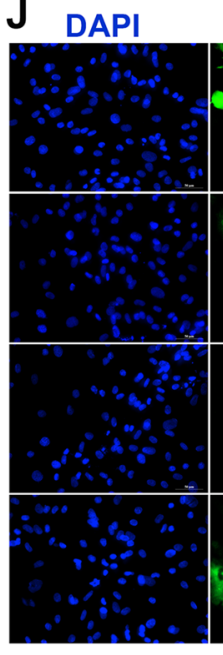

OE

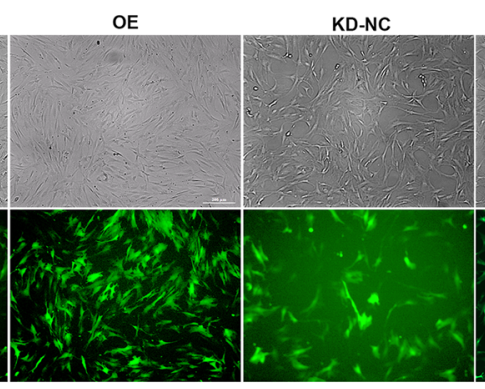

KD
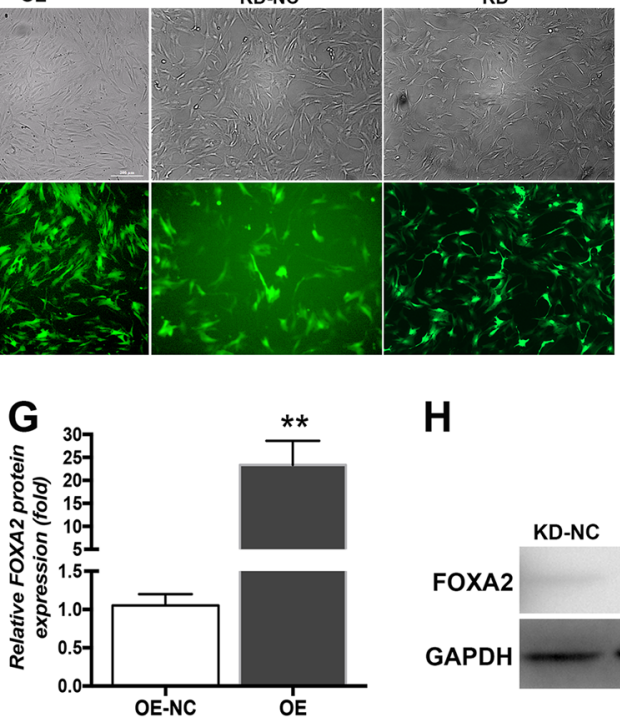

H

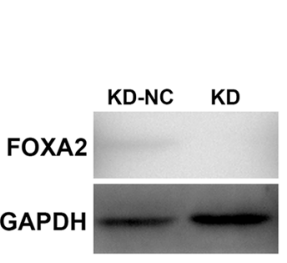

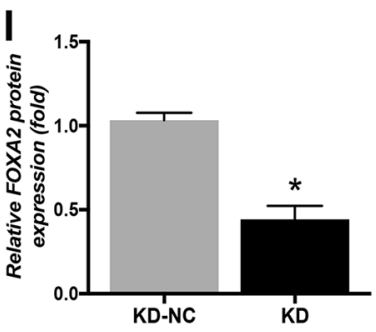

GFP

FOXA2

Merge

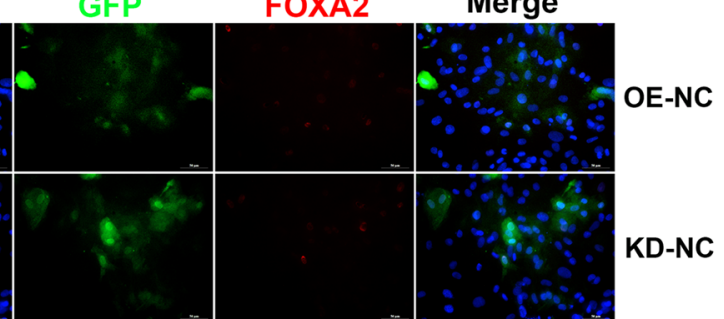

K
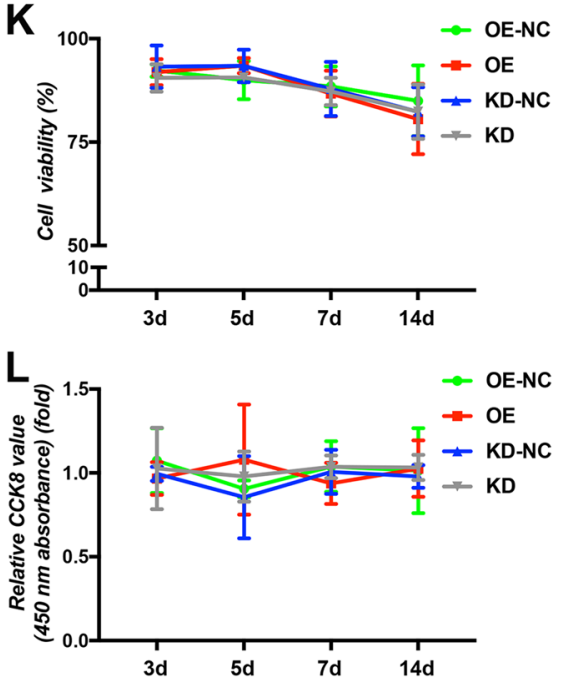

Fig. 1 Verification of FOXA2 overexpression and knockdown in BMSCs. a FOXA2 mRNA levels were decreased in differentiated BMSCs. b FOXA2 protein levels were decreased in differentiated BMSCs. $\mathbf{c}$ Relative quantitative comparison of western blot analyses mentioned in (b). Data are expressed as the mean $\pm S D$. ${ }^{*} P<0.05$ vs. undifferentiated BMSCs. d rBMSCs after lentiviral transfection and puromycin screening were observed under a normal microscope and a fluorescence microscope. Scale bars, $200 \mu \mathrm{m}$. e FOXA2 mRNA levels were significantly up- or downregulated relative to the negativecontrol groups. f FOXA2 protein levels were significantly upregulated relative to the negative control group using FOXA2-overexpressing-lentiviral particles. $\mathbf{g}$ Relative quantitative comparison of western blot analyses mentioned in (f). $\mathbf{h}$ FOXA2 protein levels were significantly downregulated relative to the negative control group using FOXA2-knockdown lentiviral particles. i Relative quantitative comparison of western blot analyses mentioned in (h). j IF analysis confirmed the successful up- or downregulation of FOXA2 protein levels. Scale bars, $50 \mu \mathrm{m}$. $\mathbf{k}$ BMSC proliferation was identified by Trypan blue staining. I BMSC proliferation was identified by the CCK-8 assay. Data are expressed as the mean \pm SD. ${ }^{*} P<0.05$ vs. OE-NC. KD knockdown of FOXA2, KD-NC negative control of FOXA2 knockdown, OE overexpression of FOXA2, OE-NC negative control of FOXA2 overexpression 


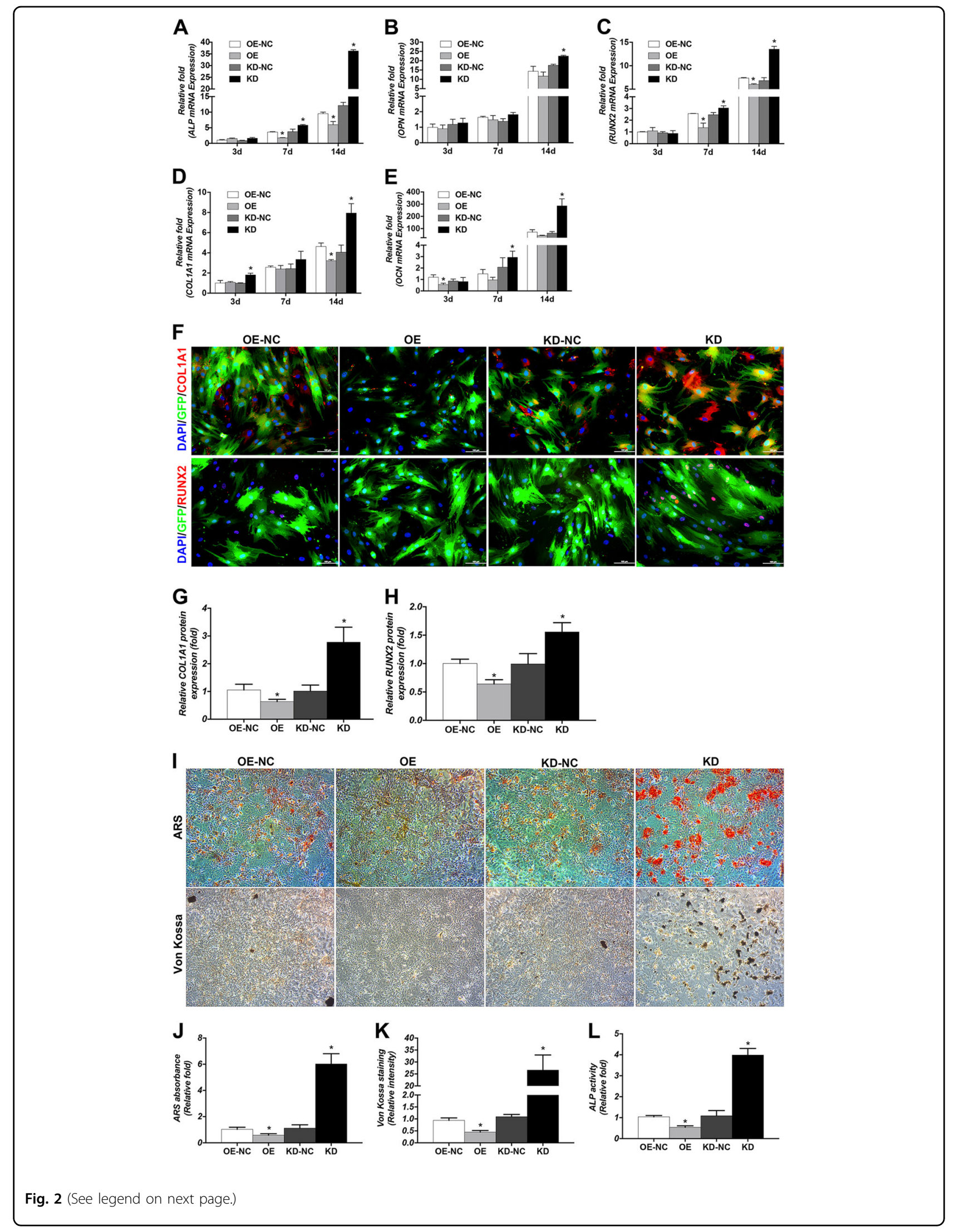


(see figure on previous page)

Fig. 2 Effects of FOXA2 on osteogenic differentiation of BMSCs. a-e Relative mRNA expression of osteospecific genes (ALP, OPN, RUNX2, COL1A1 and OCN) on d 3, 7 and 14 of osteogenesis. The mRNA expression levels were normalised to that of 185 ribosomal RNA. $\mathbf{f}$ Relative expression of osteospecific proteins (RUNX2 and COL1A1) (red) determined by immunofluorescence on day 3 of osteogenesis. Nuclei were counterstained with DAPI (blue). Scale bars, $100 \mu \mathrm{m}$. $\mathbf{g}$ Relative quantitative analysis of COL1A1 immunofluorescence staining. $\mathbf{h}$ Relative quantitative analysis of RUNX2 immunofluorescence staining. i Alizarin red staining on day 10 of osteogenic differentiation. Scale bars, $200 \mu$ m. von Kossa staining on day 10 of osteogenic differentiation. Scale bars, $500 \mu \mathrm{m}$. $\mathbf{j}$ Relative quantitative analysis of ARS. $\mathbf{k}$ Relative quantitative analysis of von Kossa staining. I ALP activity detection on day 3 of osteogenic differentiation. Data are expressed as the mean \pm SD. KD knockdown of FOXA2, KD-NC negative control of FOXA2 knockdown, OE overexpression of FOXA2, OE-NC negative control of FOXA2 overexpression
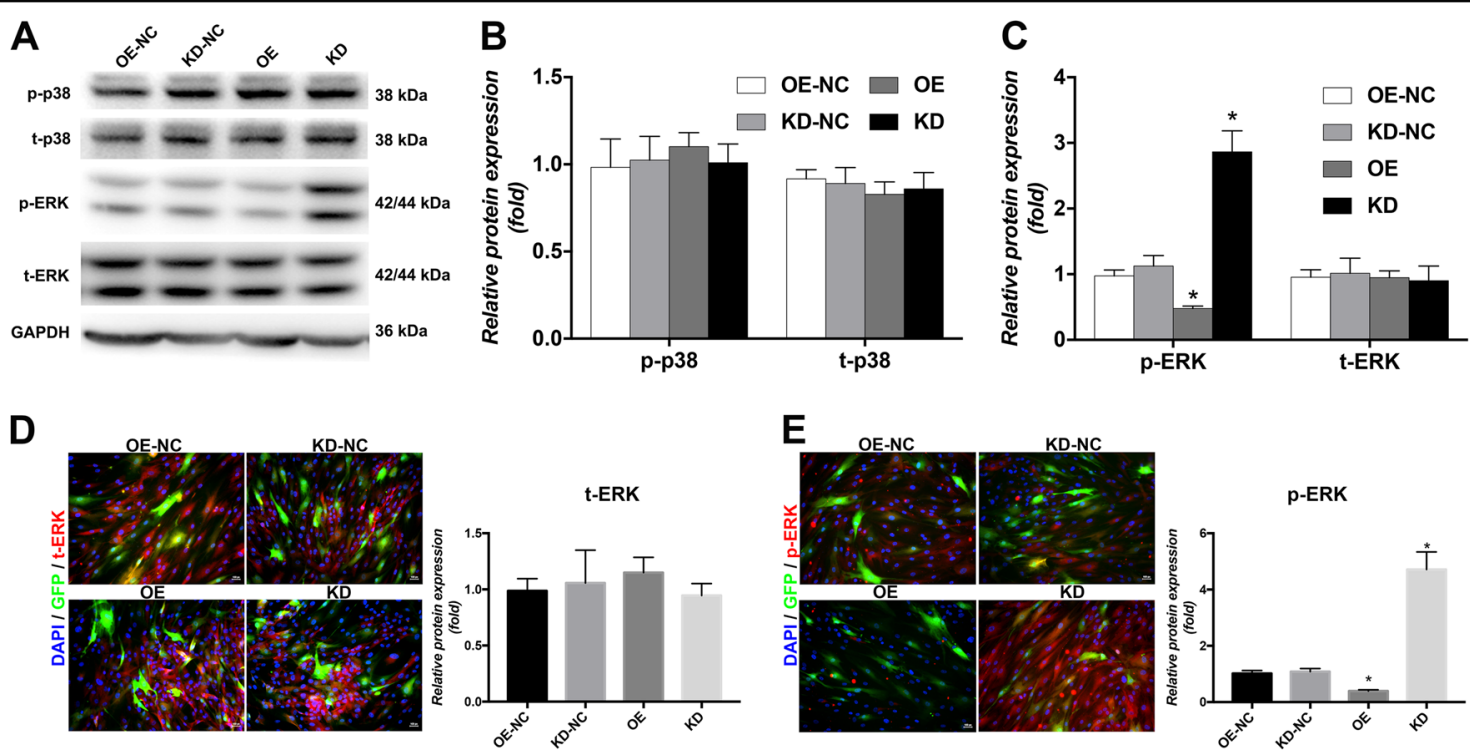

Fig. 3 FOXA2 knockdown activated the ERK signalling pathway. a Comparison of relevant expression levels of signalling pathway markers by western blot analyses between OE-NC, OE, KD-NC and KD groups. b Relative quantitative analysis of western blot analyses for p-p38 and t-p38. c Relative quantitative analysis of western blot analyses for t-ERK and p-ERK. $\mathbf{d}$ Immunofluorescence staining for t-ERK (red). e Immunofluorescence staining for $p$-ERK (red). Nuclei were counterstained with DAPI (blue). Images are magnified $\times 200$. Data are expressed as the mean \pm SD. ${ }^{*} P<0.05$ vs. OE-NC. KD knockdown of FOXA2, KD-NC negative control of FOXA2 knockdown, OE-NC negative control of FOXA2 overexpression, OE overexpression of FOXA2

\section{FOXA2-KD activated the ERK signalling pathway}

To explore the signalling pathways involved in the regulation of BMSC differentiation by FOXA2, one of the most common signalling pathways involved in osteogenesis, the mitogen-activated protein kinase (MAPK) signalling pathway was examined by western blotting. Increased expression of phosphorylated-ERK (p-ERK) was observed in the KD group on day 3 of osteogenic differentiation. Compared with the OE-NC group, the p-ERK level was significantly reduced in the $\mathrm{OE}$ group. No significant differences were found in the levels of total ERK (t-ERK), p-p38 and t-p38 among these groups (Figure 3ac). Moreover, IF confirmed increased expression of $p$-ERK accumulation in the KD group and less p-ERK in the OE group compared with the control group. No significant differences in the expression of $t$-ERK accumulation were observed (Figure 3d-e).

\section{Enhanced osteogenic differentiation of BMSCs due to FOXA2-KD was partially reduced by the addition of ERK signalling inhibitors}

To verify the involvement of the ERK signalling pathway, we evaluated the inhibitory effects of this pathway on osteogenesis in the KD group. The appropriate concentration of U0126 used to efficiently inhibit p-ERK levels was screened by western blotting. After the addition of U0126 for $24 \mathrm{~h}$, both p-ERK and t-ERK levels were examined. The level of p-ERK was significantly inhibited following treatment with at least $12.5 \mu \mathrm{M}$ (Fig. 4a). Thus, we chose $12.5 \mu \mathrm{M}$ U0126 for subsequent experiments. Following the addition of U0126 for 3 days, the level of pERK was significantly decreased compared with the level in FOXA2 knocked down rBMSCs without the inhibitor (Fig. 4b). Moreover, inhibition of ERK partially reversed the increase in osteogenesis of rBMSCs, as indicated by 


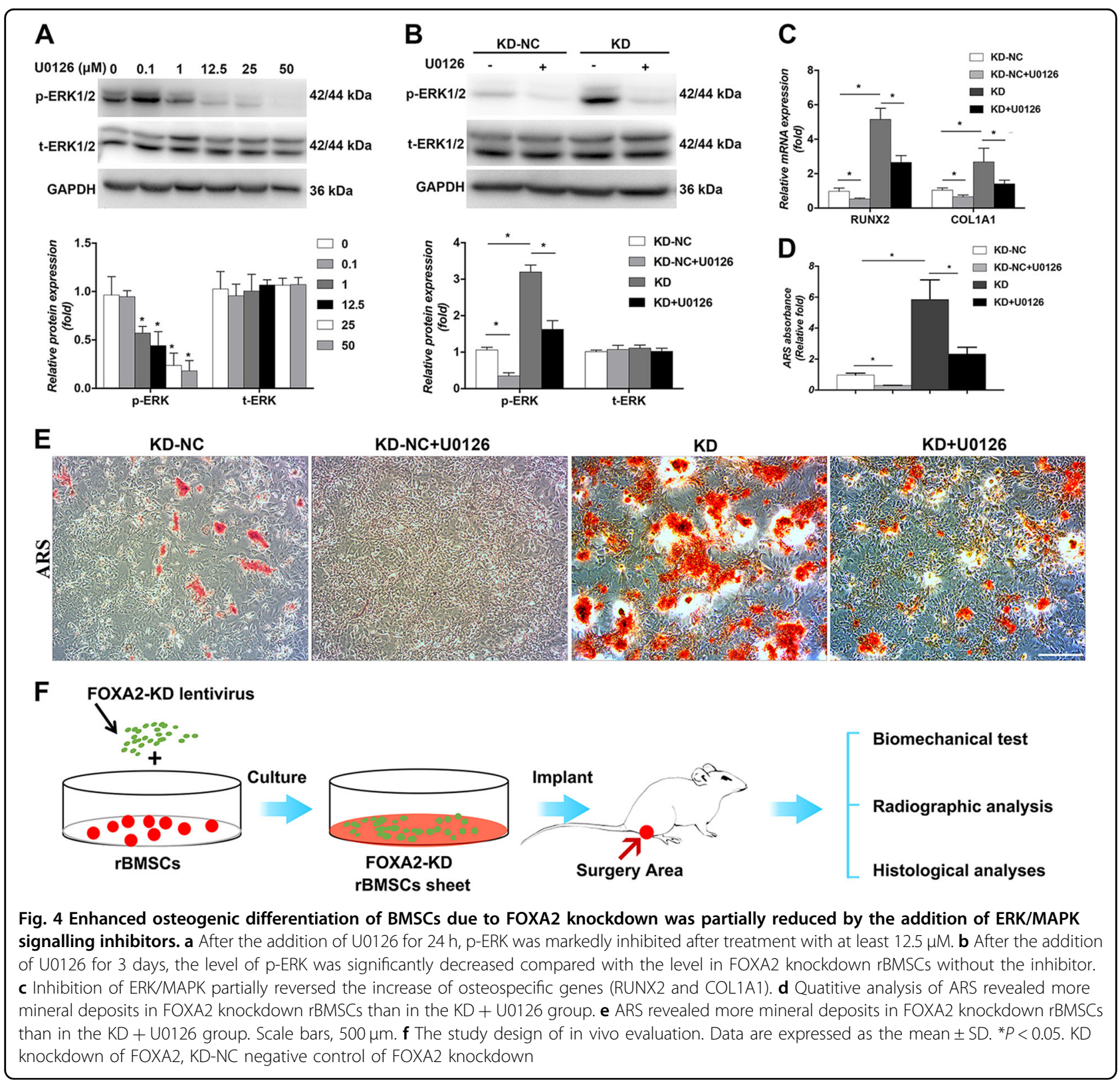

the expression of osteospecific genes (RUNX2 and COL1A1) (Fig. 4c). In addition, ARS revealed more mineral deposits in FOXA2-KD rBMSCs than in the KD + U0126 group (Fig. 4d, e).

\section{rBMSC sheets with knocked down FOXA2 accelerated bone fracture healing in a rat tibial defect model}

To further evaluate the effect of FOXA2-KD in vivo, a sheet of rBMSCs with knocked down FOXA2 was used in a rat tibial defect model. The effect was confirmed by biomechanical testing, and radiographic and histological analysis (Fig. 4f). The results of biomechanical analyses indicated that treatment with rBMSC sheets significantly increased the ultimate force and stiffness when compared with the blank group. Furthermore, a larger ultimate force and greater stiffness were found in the KD group than in the KD-NC group (Fig. 5a). Radiographs taken at 6 weeks postoperatively showed that the cortical defect was clearly present in the blank group. In the KD-NC group, this gap was smaller, and more bridging callus formation was observed at the fracture site compared with the blank group. In the KD group, the gap was obscure (Fig. 5b).

The results of micro-computed tomography $(\mu \mathrm{CT})$ analyses showed a significant increase in the bone volume fraction (BV/TV) and trabecular number (Tb.N) in KD$\mathrm{NC}$ and KD groups compared with the blank group. The increase was significantly greater in the KD group than the KD-NC group (Fig. 5c). The results of three- 

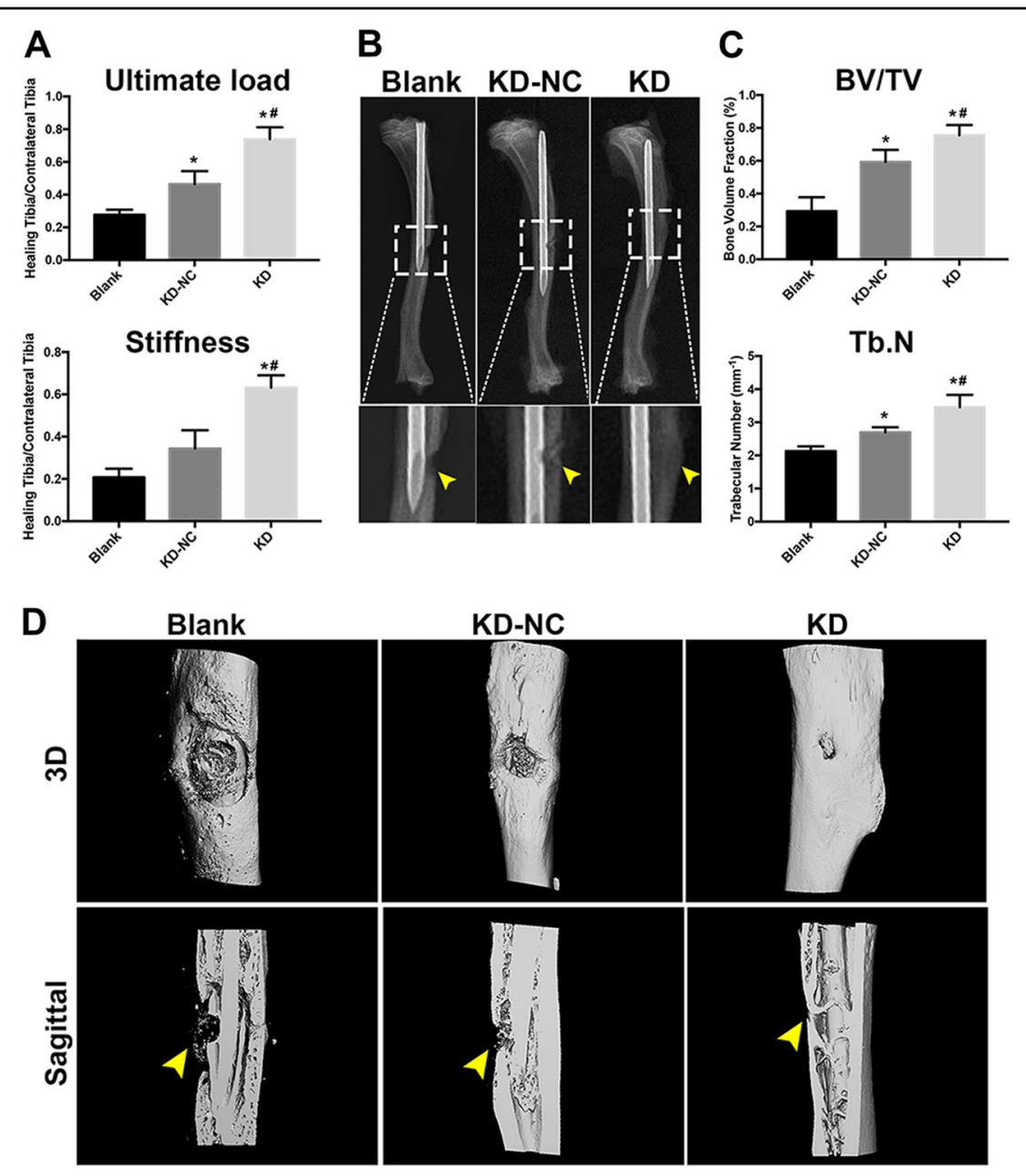

E

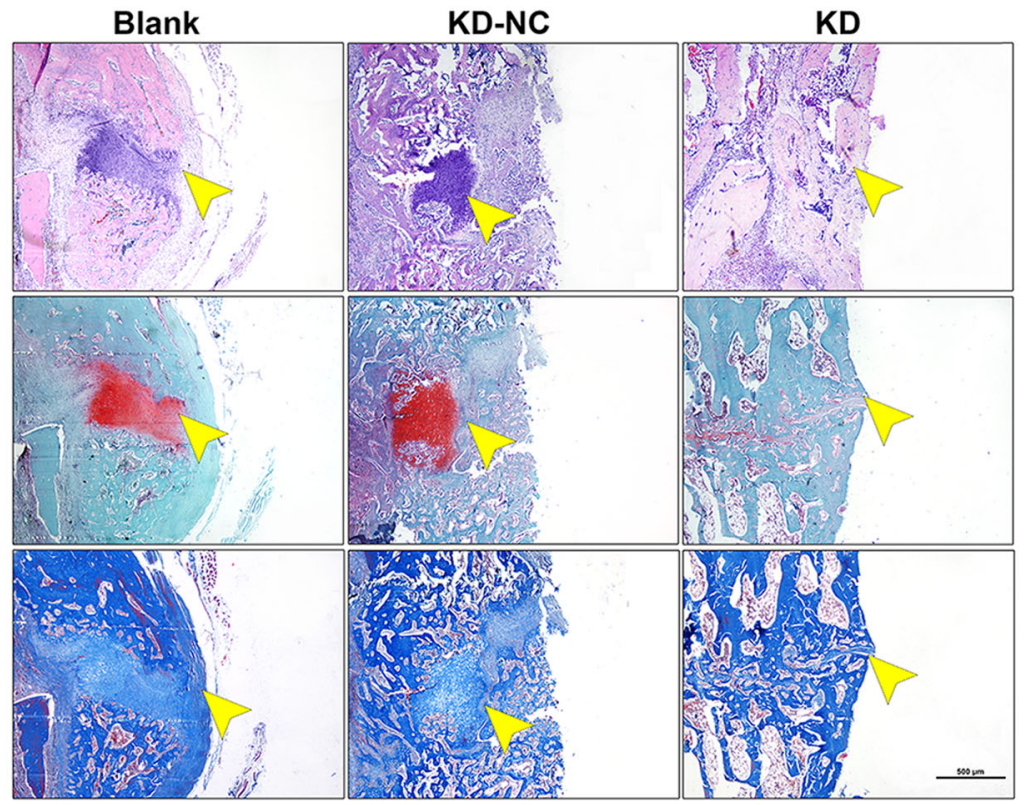

Fig. 5 (See legend on next page.) 
(see figure on previous page)

Fig. 5 A sheet of rBMSCs with FOXA2 knockdown accelerated bone fracture healing in a rat tibial defect model. a The results of biomechanical analysis indicated that treatment with rBMSCs with FOXA2 knockdown markedly increased the ultimate force and stiffness when compared with the blank group and KD-NC group. b Radiographic analysis taken at 8 weeks postoperatively. c Quatitive $\mu C T$ analyses of BV/TV and Tb.N. $\mathbf{d}$ 3D construction images of $\mu \mathrm{CT}$ analyses. $\mathbf{d}$ Histological evaluation including HE, Safranin $\mathrm{O}$ and fast green, and Masson staining of the defect area at 8 weeks after surgery in each group showed that the defects in the blank group were filled with fibrous tissue and a few chondrocytes without bridging bone formation, while a thick callus consisting of newly formed woven bone tissue was observed in the defect area in the KD-NC group. In the FOXA2 knocked down rBMSC group, the defect sites were almost sealed, and remodelling of the callus was more complete. Yellow arrow: the defect area. Scale bars, $500 \mu \mathrm{m}$. Data are expressed as the mean \pm SD. ${ }^{*} P<0.05$ vs. blank group; ${ }^{\#} P<0.05$ vs. KD-NC. KD knockdown of FOXA2, KD-NC negative control of FOXA2 knockdown

dimensional reconstruction of the $\mu \mathrm{CT}$ results showed that the largest defect appeared in the blank group, whereas a smaller defect was observed in the KD-NC group. In the KD group, the defect almost disappeared, and more bridging callus formation was observed, when compared with those of the other two groups (Fig. 5d).

Histological analyses, including hematoxylin and eosin (HE), Safranin O/Fast Green and Masson's trichrome staining, showed that the defects in the blank group were filled with fibrous tissue and a few chondrocytes; no bridging bone formation at the defect site was observed. In the KD-NC group, a thick callus consisting of newly formed woven bone tissue was observed in the defect area. In the FOXA2 knocked down rBMSC group, the defect sites were almost sealed, and remodelling of the callus was more complete, indicating bone healing of the defect (Fig. 5e).

\section{Discussion}

In the present study we showed that FOXA2, a member of the forkhead/winged-helix family of transcription factors, which has important roles in cell differentiation, development and tissue homoeostasis, acted as an osteogenic differentiation-suppressing gene in MSCs. We found that endogenous expression of FOXA2 was downregulated in rBMSCs during osteogenesis. We therefore used a FOXA2-KD or OE strategy to promote or inhibit osteogenic differentiation of MSCs and found that FOXA2-KD accelerated osteogenesis of rBMSCs via activation of the ERK signalling pathway in vitro. Moreover, a rBMSC sheet with knocked down FOXA2 accelerated bone fracture healing in a rat bone defect model. These findings indicated that FOXA2-KD enhanced osteogenesis of rBMSCs, at least partially by activation of the ERK signalling pathway.

This study emphasised a novel and pivotal role for FOXA2 in regulating the osteogenic differentiation of BMSCs. The Fox protein family consists of 17 subfamilies of transcription factors that display a winged-helix structure in the DNA-binding region. As an important transcription factor, studies of FOXA2 have mainly focused on its regulation of hepatocyte differentiation and pulmonary morphogenesis. A previous study reported that lentivirus-mediated OE of FOXA2 induced rBMSCs into hepatocytes, when tested in vitro ${ }^{26}$. Glucose metabolism is being increasingly accepted as a predominant factor that influences bone ageing and the differentiation potency of $\mathrm{MSCs}^{27}$. Wolfrum et $\mathrm{al}^{28}$ reported that FOXA2 is a critical transcription factor in regulating both lipid and glucose metabolism by modulating expression of various genes, including fatty acid synthase, scd-1, and glucose 6-phosphatase in the liver. Recently, Ionescu et al. $^{29}$, using mice engineered to lack expression of FoxA2, reported that FOXA2 directly modified collagen X expression and promoted chondrocyte hypertrophy, which further supported the connection between FoxA2 and bone development. Wnt 3 and WNT7b, which are closely related with bone development, have also been found to be directly regulated by FOXA $2^{18,19}$. To some extent, these previous studies further supported our findings that FOXA2 plays an important role in osteogenesis.

Based on previous studies ${ }^{30,31}$, several lineage-specific regulators of osteogenic differentiation have been identified using various approaches such genetic approaches. For examples, regulators that upregulate the expression Wnt3a/Wnt10b ${ }^{32}, \quad$ BMP2 $^{33}, \quad$ Nell-1 ${ }^{34}, \quad$ Msx2 $^{35}$ and $\mathrm{SPARC}^{36}$, etc. have been proved to promote the determination of BMSCs into osteoblasts. It is interesting to notice that data involving other protein families such as SFRP2, NURR1, heparin-binding EGF-like growth factor (HB-EGF) and RHEB in the literature reported similar results to the results of this study ${ }^{37-40}$. Jin et al..$^{37}$ reported that SFRP2 enhances the osteogenic differentiation of apical papilla stem cells by antagonising the canonical WNT pathway. Di Benedetto et al. $^{38}$ reported that downregulation of NURR1 strongly prompted the differentiation of dental pulp stem cells towards the osteoblastogenesis process. Using human adipose-derived MSCs, Ashraf et al. ${ }^{40}$ demonstrated that RHEB increased the osteogenesis via the upregulation of Runx2. In addition, microRNAs were also found to be involved in regulating the osteogenic differentiation. Yu et al. ${ }^{39}$ showed that forced expression of HB-EGF or treatment with HB- 
EGF was capable of reducing osteogenic differentiation of $\mathrm{C} 2 \mathrm{C} 12$ cells, whereas upregulation of miR-1192 enhanced Runx2-induced osteogenesis ${ }^{39}$. In summary, multiple regulators of certain cell lineages are involved in osteogenic differentiation. The effects of FOXA2 on osteogenic differentiation found in this study may also be related with the complicated interaction with other protein families or microRNAs, which indicates that molecular switches of these regulators may prove to be ideal "druggable" targets for future clinical intervention aiming at enhancing bone formation.

MAPKs, which consist of ERK, c-jun $\mathrm{NH}_{2}$-terminal kinase and p38 MAPK ${ }^{41}$, are important signal transducers in the regulation of osteogenic differentiation of MSCs and bone metabolism ${ }^{42}$. Among them, the ERK signalling pathway, which is activated during osteogenic differentiation, is of vital importance. A majority of the secreted ligands that modulate osteoblast activity appear to act in part through the ERK pathway ${ }^{43}$. In addition, runx2 and osterix are strongly regulated through ERK phosphorylation $^{44}$. Mice with deletions of Erk1 and Erk2 display dramatically reduced bone mineralisation, demonstrating the important role of ERK in osteoblast mineralisation ${ }^{45}$. With regard to the relationship between FOXA2 and ERK signalling, An et al. ${ }^{46}$ reported that valproic acid increased the expression of FOXA2 in MSCs by activating signal transduction of ERK. Our western blotting and IF analyses showed that the ERK signalling pathway was strongly related to FOXA2-KD-promoted osteogenesis. In addition, to confirm our findings, we also examined whether blocking the ERK signalling pathway decreased the effect of FOXA2-KD on rMSC osteogenesis, using U0126. Treatment with U0126 blocked ERK1/2 phosphorylation and significantly decreased runx2, col1a1 activity and mineralised nodule formation in the FOXA2KD groups. These results indicated that ERK1/2 had a crucial role during FOXA2-KD-induced osteogenesis in rMSCs.

To the best of our knowledge, this is the first study to characterise the effects of FOXA2 on MSC osteogenic differentiation. However, some limitations of our study should be noted. First, although we investigated the role of FOXA2 on osteogenesis, the role of FOXA2 on another important process, osteoclastogenesis, was not studied. Future studies are needed. Second, the molecular mechanism of osteogenesis is complicated; although we indicated that FOXA2 mediated the ERK signalling pathway to regulate the osteogenic differentiation of BMSCs, other signalling pathways may also be involved. The interaction between FOXA2 and the osteogenic differentiation-related genes, also remains to be uncovered in further studies. Third, the nuclear and cytoplasmic localisation of FOXA2, together with its phosphorylation status, were not studied, which may decrease the robustness of the conclusions of this study. Nonetheless, this study used both $\mathrm{OE}$ and $\mathrm{KD}$ to explore the role of FOXA2, which provided useful insight into the molecular mechanism of bone development and the potential effects of FOXA2 in regulating the osteogenic differentiation of BMSCs. Future studies using genetically engineered mice or large animals are required to further verify our findings.

\section{Conclusion}

FOXA2-KD enhanced osteogenic differentiation of rBMSCs, partly via activation of the ERK signalling pathway.

\section{Materials and Methods \\ Cell culture and reagents}

rBMSCs were purchased from Cyagen Biosciences (RASMX-01001, Guangzhou, China), which were cultured in complete rBMSC growth medium (RASMX90011, Cyagen Biosciences, Inc., Guangzhou, China). Adherent cells were passaged after reaching 80\%-90\% confluence. Cells from passages 3-9 were used in subsequent experiments. U0126 was purchased from Cell Signalling Technology (Shanghai, China).

\section{Lentiviral packaging and cell infection}

All the lentiviral particles were prepared by Cyagen Biosciences. Three oligonucleotides (5'-GACGCTG AGCGAGATCTAT-3', 5'-GCGCTTCAAGTGTGAG AAC-3' and 5'-GCTGCAGACACTTCCTACT-3'), encoding a 19 nt-long short-hairpin RNA (shRNA) against rat FOXA2, were designed and the most effective one among them was chosen for the following experiments. A scrambled shRNA sequence $\left(5^{\prime}\right.$-TTCTC CGAACGTGTCACGT-3'), exhibiting no homology to the rat sequence database, was used as a negative control [FOXA2-KD control group (KD-NC)]. The oligonucleotides were phosphorylated, annealed and cloned into the pLVX-shRNA vector. The resulting vectors, designated pLVX-rat-shFOXA2 and pLVX-rat-shFOXA2-Control, were subsequently verified by sequencing. Lentivirus overexpressing FOXA2 (lenti-FOXA2) particles and lentiviral GFP particles (lenti-control) were prepared by Cyagen Biosciences. The lentiviral GFP particles were used as control group [FOXA2 OE control group $(\mathrm{OE}-\mathrm{NC})]$ in this study. For retroviral OE of FOXA2, the full-length cDNA of human FOXA2 (NM_012743.1) was obtained by PCR. Next, FOXA2 cDNA was subcloned into the BamHI and XhoI sites of the CMV-MCSEGFPIRES retroviral vector (Cyagen Biosciences).

For infections, rBMSCs were incubated with lentiviral particles and polybrene $(5 \mu \mathrm{g} / \mathrm{mL})$ in growth medium. After about $24 \mathrm{~h}$, the infection medium was discarded. After 3 days, the cells were screened using puromycin 
$(4 \mu \mathrm{g} / \mathrm{mL})$ and then passaged for use in subsequent experiments. Transduction efficiency was monitored by GFP fluorescence. The expression of FOXA2 was quantified by quantitative real-time PCR and western blot analyses.

\section{Trypan blue staining}

After infection of lentiviral particles, rBMSCs were collected on d 3, 5, 7 and 14. Cell viability was determined using trypan blue staining by an Automated Cell Counter (Countess; Thermo Fisher Scientific, Waltham, MA, USA) as previously described ${ }^{47}$.

\section{Cell Counting Kit-8}

To assess the effect of FOXA2 OE and KD on the proliferation of rBMSCs, the cells were seeded into a 96well plate (5000/well) and allowed to adhere for $24 \mathrm{~h}$. Then the medium was removed and the cells were treated with 10\% CCK-8 (Dojindo, Kumamoto, Japan) in $150 \mu \mathrm{L}$ Dulbecco's modified Eagle's medium (DMEM) without fetal bovine srum (FBS) for $3 \mathrm{~h}$ at $37^{\circ} \mathrm{C}$. Absorbance at $450 \mathrm{~nm}$ was measured using a microplate reader (ELX808; BioTek, Winooski, VT, USA).

\section{Osteogenic differentiation protocol}

For the osteogenic differentiation, rBMSCs were first cultured in growth medium (RASMX-90011, Cyagen Biosciences, Inc.) in 6- or 12-well cell culture plates (Corning, Shanghai, China), at a density of $3 \times 10^{4} / \mathrm{cm}^{2}$ and incubated for $48 \mathrm{~h}$ at $37^{\circ} \mathrm{C}$ under $5 \% \mathrm{CO}_{2}$. The rBMSCs were subsequently cultured in osteogenic induction medium (low-sugar DMEM; 10\% FBS (1495527; Gibco, Waltham, MA, USA), $100 \mathrm{nM}$ dexamethasone, $100 \mathrm{IU} / \mathrm{ml}$ penicillin/streptomycin, $10 \mathrm{mM}$ $\beta$-glycerophosphate and $0.2 \mathrm{mM}$ ascorbic acid), which were then maintained by the addition of fresh osteogenic induction medium every $2-3$ days.

\section{Measurement of ALP activity and ALP staining}

For the measurement of ALP activity, osteogenic differentiated cells were lysed in radioimmunoprecipitation assay (RIPA, Beyotime, Shanghai, China) lysis buffer consisting of $20 \mathrm{mM}$ Tris- $\mathrm{HCl}$ (pH 7.5), $150 \mathrm{mM} \mathrm{NaCl}$ and $1 \%$ Triton X-100. Then ALP activity was determined using an ALP Activity Assay (Beyotime) according to the manufacturer's instructions. Briefly, $10 \mu \mathrm{l}$ lysate was incubated with $90 \mu \mathrm{l}$ fresh solution containing pnitrophenyl phosphate substrate at $37^{\circ} \mathrm{C}$ for $30 \mathrm{~min}$. Then, $100 \mu \mathrm{l} 0.5 \mathrm{~N} \mathrm{NaOH}$ was used to stop the reaction. The absorbance was measured at $405 / 650 \mathrm{~nm}$ on a microplate reader (ELX808; BioTek). For ALP staining, cells were fixed with $4 \%$ paraformaldehyde (Sigma, Shanghai, China) for $15 \mathrm{~min}$ at room temperature and washed three times with phosphate-buffered saline (PBS).
Cells were then stained using a BCIP/NBT ALP Color Development Kit (Beyotime, Shanghai, China).

\section{ARS staining}

For the measurement of mineral deposition after the induction of osteogenic differentiation, ARS was used. Cells were fixed in $4 \%$ paraformaldehyde (Sigma) for $15 \mathrm{~min}$ at room temperature and then washed three times with PBS. Then, $1 \%$ solution of ARS kit (Cyagen Biosciences) was added and incubated for $30 \mathrm{~min}$ at room temperature, followed by rinsing with PBS. The solution was collected and the absorbance at $560 \mathrm{~nm}$ of $200 \mu \mathrm{l}$ solution of stained cells in 96-well plates was read using a microplate reader (ELX808; BioTek). The readings were normalised to the total protein concentrations.

\section{Von Kossa staining}

For von Kossa staining, osteogenic differentiated cells were fixed with $4 \%$ paraformaldehyde (Sigma) for $15 \mathrm{~min}$ at room temperature and washed three times with PBS. Next, cells were incubated with a $5 \%$ silver nitrate solution and were exposed to UV radiation for $30 \mathrm{~min}$. After that, $5 \%$ sodium thiosulfate was added for $5 \mathrm{~min}$, to remove nonspecific staining.

\section{Immunofluorescence}

Cells were cultured in a 12-well plate, and FOXA2, RUNX2, COL1A1, t-ERK and p-ERK were detected using a fluorescence microscope (EU5888; Leica, Wetzlar, Germany). Briefly, cells were fixed in $4 \%$ paraformaldehyde (Sigma) for $15 \mathrm{~min}$ at room temperature, permeabilized and blocked for $30 \mathrm{~min}$ in $0.05 \%$ Triton X-100 and $5 \%$ bovine serum albumin (BSA). Fixed cells were washed three times with PBS and incubated at $4{ }^{\circ} \mathrm{C}$ overnight with anti-FOXA2 (1:400; Cell Signalling Technology), RUNX2 (1:1600; Cell Signalling Technology), COL1A1 (1:500; Abcam, Shanghai, China), t-ERK (1:800; Cell Signalling Technology) or p-ERK (1:200; Cell Signalling Technology). Cells were incubated with a fluorescence-conjugated secondary antibody (Beyotime) at room temperature for $2 \mathrm{~h}$ and nuclei were stained with 4',6-diamidino-2-phenylindole (KeyGen Biotech, Nanjing, China) for $4 \mathrm{~min}$. Samples were then observed and photographed under a fluorescence microscope (Leica).

\section{RNA isolation and qPCR}

Total cellular RNA was isolated using RNAiso reagent (Takara, Dalian, China) and quantified by measuring the absorbance at $260 \mathrm{~nm}$ (NanoDrop 2000; Thermo Fisher Scientific). Total RNA ( $\leq 1000 \mathrm{ng}$ ) was then reversetranscribed into cDNA in a reaction volume of $10 \mu \mathrm{l}$ using a Double-Strand cDNA Synthesis Kit (Takara). All gene transcripts were quantified by qPCR using the Power SYBR $^{\circ}$ Green PCR Master Mix (Takara) on the ABI 
Table 1 Sequences of primers for quantitative real-time PCR

\begin{tabular}{lll}
\hline Gene & Reverse $\left(\mathbf{5}^{\prime} \mathbf{-} \mathbf{3}^{\prime}\right)$ & Reverse $\left(\mathbf{3}^{\prime} \mathbf{-} \mathbf{5}^{\prime} \mathbf{)}\right.$ \\
\hline FOXA2 & CACGGCTCCCAGCATACTT & CACGGCTCCCAGCATACTTT \\
ALP & GCCGGCCCAAGAGAGAA & CCGATGGGACCGTGGT \\
RUNX2 & CAGCAGAGGCATTTCGTAGCT & CATCGGTGGTACTAAC \\
COL1A1 & CTGGATCATATTGCACA & GAGCTGCCCTGCACTGGGTG \\
OCN & TGGCCCCAGACCTCTTCCCG & TGGCCCCAGACCTCTTCCCG \\
OPN & CAGGCTGGCTTTGGACT & CAGGCTGGCTTGGAACT \\
18S & TTGACGGAAGGGCACCA & TTGACGGAAGGGCACCA \\
\hline
\end{tabular}

StepOnePlus System (Applied Biosystems, Warrington, UK). $18 \mathrm{~S}$ was used as a housekeeping gene. The mRNAs of the target genes and the housekeeping gene were quantified in separate tubes. All primers used in this study were synthesised by Sangon Biotech (Shanghai, China), the primer sequences of which were shown in Table 1 . The cycle conditions of qPCR were set as follows: $95^{\circ} \mathrm{C}$ for $30 \mathrm{~s}$ and then 42 cycles of $95^{\circ} \mathrm{C}$ for $5 \mathrm{~s}$ and $60^{\circ} \mathrm{C}$ for $30 \mathrm{~s}$. The $2^{-\triangle \triangle \mathrm{Ct}}$ method was used to calculate the relative expression levels of target genes.

\section{Western blot analysis}

Protein extracts from cells were prepared in RIPA lysis buffer supplemented with a proteasome inhibitor (Beyotime, Haimen, China). Total proteins were separated by $10 \%$ SDS-polyacrylamide gel electrophoresis and then transferred to a polyvinylidene difluoride membrane (Millipore, Shanghai, China). After blocking in 5\% BSA for $1 \mathrm{~h}$ at room temperature, the membranes were incubated overnight at $4{ }^{\circ} \mathrm{C}$ with antibodies specific to GAPDH (1:2000, Cell Signalling Technology), FOXA2 (1:1000; Cell Signalling Technology), RUNX2 (1:1000; Cell Signalling), COL1A1 (1:1000; Abcam), t-ERK (1:1000; Cell Signalling Technology) or p-ERK (1:2000; Cell Signalling Technology). Horseradish peroxidase-conjugated goat anti-rabbit IgG (1:5000, Boster Biologic Technology, Wuhan, China) was used as a secondary antibody for $2 \mathrm{~h}$ at room temperature. The immunoreactive bands were visualised using an enhanced chemiluminescent detection reagent (Millipore). Signal intensity was quantified using a Bio-Rad XRS chemiluminescence detection system (Bio-Rad, Hercules, CA, USA).

\section{Cell sheet preparation}

The protocol of cell sheet preparation was according to our previously reported studies ${ }^{48,49}$. Briefly, confluent cells $\left(1 \times 10^{5} / \mathrm{cm}^{2}\right)$ in flasks were cultured in MSC growth medium with the addition of vitamin $C(20 \mu \mathrm{g} / \mathrm{mL})$ for 2 weeks to form a sheet of rBMSCs. Cells were then rinsed twice with PBS, and then detached intact from the substratum as cell sheets using a scraper.

\section{In vivo evaluation in animals}

All Sprague-Dawley (SD) rats were supplied by the Academy of Medical Sciences of Zhejiang Province. The protocol of all animal experiments was approved by the Institutional Animal Care and Use Committee of the Second Affiliated Hospital, School of Medicine, Zhejiang University. All animal experiments were performed in accordance with the Animal Care and Use Committee guidelines of Zhejiang province, strictly following the guidelines for the care and use of laboratory animals. The animals had free access to food and water and were kept in a pathogen-free animal room.

In total, 30 male SD rats weighing (8-week-old, weighing 250-300 g) were used to establish a rat tibial defect model. The rats were divided randomly and evenly into three groups: a blank group, an KD-NC (negative-control group of rBMSCs with FOXA2-KD) group and an KD (rBMSCs with FOXA2-KD) group ( $n=10$ per group). All surgical procedures were performed by two experienced orthopaedic surgeons. The tibial defect model was established as reported previously ${ }^{50,51}$. Briefly, rats were anaesthetised intraperitoneally with $0.3 \%$ pentobarbital sodium (Sigma) at $30 \mathrm{mg} / \mathrm{kg}$ body weight. After anaesthesia, an incision was made below the knee, which was followed by an intramedullary needle $(1.2 \mathrm{~mm}$-diameter stainless steel syringe needle) being inserted inside the medullary canal of the tibia for fixation ${ }^{52}$. A $1.5 \mathrm{~mm}$ diameter tibial defect was made in all SD rats approximately $1 \mathrm{~cm}$ from the proximal tibial growth plate by a hollow drill ${ }^{51-53}$. The same leg was used for each group. As is introduced above, cell sheets with FOXA2$\mathrm{KD}$ and the negative controls were prepared with confluent cells $\left(1 \times 10^{5} / \mathrm{cm}^{2}\right)$ in flasks for 2 weeks before use. The details of cell sheet implantation were introduced in our previous studies ${ }^{48,49,54}$. In brief, in the blank group $(n=10)$, nothing was grafted onto the tibial defect site; in the KD-NC group, a sheet of KD-NC rBMSCs were used to fill the defects and wrap around the defect areas; in the KD group, a sheet of rBMSCs with FOXA2$\mathrm{KD}$ was implanted into the defects and wrap around the defect areas (Fig. 4f).

All of the rats were sacrificed in a $\mathrm{CO}_{2}$ chamber at 6 weeks postoperatively. Specimens of the experimental and the contralateral intact tibias were collected for biomechanical testing, radiographic analysis, and histological analysis. For biomechanical testing, specimens $(n=5$ each group) were immediately frozen at $-80^{\circ} \mathrm{C}$ and thawed overnight at $4{ }^{\circ} \mathrm{C}$ for testing. For radiographic and histological analysis, specimens ( $n=5$ each group) were fixed in $4 \%$ paraformaldehyde (Sigma) for $48 \mathrm{~h}$ at room temperature and kept in PBS. 


\section{Biomechanical testing}

To evaluate the function of the experimental tibia, a three-point bending biomechanical test was performed as previously described ${ }^{48,55}$. Both the experimental and the contralateral intact tibias were tested compared using a Zwick/Roell 2.5 material testing system (Zwick, Ulm, Germany). All the tibia samples were kept moist with normal saline during biomechanical testing. A preloaded of $2 \mathrm{~N}$ at a speed of $0.2 \mathrm{~mm} / \mathrm{s}$ was applied to adapt for $10 \mathrm{~s}$. Thereafter, tibia samples from rats were tested using a vertical pushing load until failure at a constant speed of $1.0 \mathrm{~mm} / \mathrm{s}$. The time, force, and displacement until failure were recorded. The ultimate load, stiffness and absorbed energy were determined and calculated based on the forcedisplacement curve,

\section{Radiographic analysis}

To evaluate callus formation and bridging bone formation at the fracture sites 6 weeks postoperatively, radiographs ( $n=5$ for each group) were taken using a dual-track molybdenum/rhodium + Mo target mammography machine $(22 \mathrm{KV}, 250 \mathrm{mAS}$; GE, Fairfield, CT, USA). For the $\mu \mathrm{CT}$ evaluation, tibia samples were scanned using a $\mu \mathrm{CT}-100$ imaging system (Scanco Medical, Brüttisellen, Switzerland) with X-ray energy settings of 70 $\mathrm{kVp}, 1024$ reconstruction matrix, $14.8 \mu \mathrm{m}$ slice thickness with an exposure time of $300 \mathrm{~ms}$. The BV/TV and Tb.N were calculated by three-dimensional standard microstructural analysis ${ }^{48,56}$.

\section{Histological evaluation}

After $\mu \mathrm{CT}$ scanning, the specimens $(n=5$ for each group) were decalcified in $10 \%$ ethylene diaminetetra acetic acid (EDTA, Sigma) with $0.1 \mathrm{M}$ PBS for more than 60 days, with a solution change once a week. Thereafter, the specimens were embedded in paraffin using standard procedures. Serial sections $(3 \mu \mathrm{m}$ thickness) were cut and mounted onto polylysine-coated glass slides, and deparaffinized. Sections were stained with HE, Safranin O/Fast Green and Masson's trichrome staining were performed separately consecutive tissue sections in accordance with our previous studies ${ }^{48,52}$. Images were obtained on a traditional light microscopy (Leica DM4000B; Leica, Solms, Germany).

\section{Statistical analysis}

Statistical analysis was performed using SPSS 19.0 software (IBM, Armonk, NY, USA). All experiments were carried out at least in triplicate, and the data are presented as means $\pm \mathrm{SD}$. To calculate the significant differences, a two-tailed Student's $t$-test was used when comparing two groups, one-way ANOVA followed by Bonferroni's post-hoc test was used when comparing more than two groups. A value of $P \leq 0.05$ was considered to indicate statistical significance.

\section{Acknowledgements}

This work was supported by grants from the National Natural Science Foundation of China (numbers 81572124, 81672147 and 81271973) and Zhejiang Provincial Natural Science Foundation of China (LY15H060003).

\section{Author details}

${ }^{1}$ Department of Orthopedic Surgery, The Second Affiliated Hospital, School of Medicine, Zhejiang University, No. 88, Jiefang Road, Hangzhou 310009, China. ${ }^{2}$ Orthopedics Research Institute of Zhejiang University, No. 88, Jiefang Road, Hangzhou 310009, China. ${ }^{3}$ Department of Rheumatology, The Second

Affiliated Hospital, School of Medicine, Zhejiang University, Hangzhou, China

\section{Author contributions}

W.Z., R.X.H., C.Y.Y., Z.J.P., M.C. and E.M.C.: conception and design. C.Y.Y., M.C., E.M.C., W.X.L., S.D.W., Q.H.D., C.W., C.H.Z., L.T., W.D.H. and K.H.: experiments and/ or data analysis. C.Y.Y., M.C. and E.M.C.: intellectual input and supervision. W.Z., R.X.H. and C.Y.Y.: article writing with contributions from other authors.

Conflict of interest

The authors declare that they have no conflict of interest.

\section{Publisher's note}

Springer Nature remains neutral with regard to jurisdictional claims in published maps and institutional affiliations.

Received: 10 May 2018 Revised: 26 June 2018 Accepted: 5 July 2018 Published online: 06 August 2018

\section{References}

1. Gomez-Barrena, E. et al. Bone fracture healing: cell therapy in delayed unions and nonunions. Bone 70, 93-101 (2015).

2. Bratton, A., Eisenberg, J., Vuchkovska, A., Roper, P. \& Callaci, J. J. The effects of episodic alcohol exposure on BMP2 signaling during tibia fracture healing. J. Orthop. Trauma 32, 288-295 (2018).

3. Mi, M. et al. Chondrocyte BMP2 signaling plays an essential role in bone fracture healing. Gene 512, 211-218 (2013).

4. Tsuij, K et al. BMP2 activity, although dispensable for bone formation, is required for the initiation of fracture healing. Nat. Genet. 38, 1424-1429 (2006).

5. Xu, M. T. et al. Diabetes mellitus affects the biomechanical function of the callus and the expression of TGF-beta1 and BMP2 in an early stage of fracture healing. Braz. J. Med. Biol. Res. 49, e4736 (2016).

6. Alwattar, B. J., Schwarzkopf, R. \& Kirsch, T. Stem cells in orthopaedics and fracture healing. Bull. Nyu. Hosp. Jt. Dis. 69, 6-10 (2011).

7. Dong, C. H. et al. The interplay of transcriptional and post-transcriptional regulation of migration of mesenchymal stem cells during early stages of bone fracture healing. Eur. Rev. Med. Pharmacol. Sci. 21, 5542-5547 (2017)

8. Han, D., Han, N., Zhang, P. \& Jiang, B. Local transplantation of osteogenic predifferentiated autologous adipose-derived mesenchymal stem cells may accelerate non-union fracture healing with limited pro-metastatic potency. Int. J. Clin. Exp. Med. 8, 1406-1410 (2015).

9. Alm, J. J. et al. Circulating plastic adherent mesenchymal stem cells in aged hip fracture patients. J. Orthop. Res. 28, 1634-1642 (2010).

10. Cheung, W. H., Chin, W. C., Wei, F. Y., Li, G. \& Leung, K. S. Applications of exogenous mesenchymal stem cells and low intensity pulsed ultrasound enhance fracture healing in rat model. Ultrasound Med. Biol. 39, 117-125 (2013).

11. Granero-Molto, F. et al. Mesenchymal stem cells expressing insulin-like growth factor-I (MSCIGF) promote fracture healing and restore new bone formation in Irs1 knockout mice: analyses of MSCIGF autocrine and paracrine regenerative effects. Stem Cells 29, 1537-1548 (2011).

12. Dilogo, I. H., Primaputra, M. R. A., Pawitan, J. A. \& Liem, I. K. Modified Masquelet technique using allogeneic umbilical cord-derived mesenchymal stem cells for infected non-union femoral shaft fracture with a $12 \mathrm{~cm}$ bone defect: a case report. Int. J. Surg. Case Rep. 34, 11-16 (2017). 
13. Fournier, M. et al. FOXA and master transcription factors recruit Mediator and Cohesin to the core transcriptional regulatory circuitry of cancer cells. Sci. Rep. 6, 34962 (2016).

14. Hannenhalli, S. \& Kaestner, K. H. The evolution of Fox genes and their role in development and disease. Nat. Rev. Genet. 10, 233-240 (2009).

15. Li, Z., Schug, J., Tuteja, G., White, P. \& Kaestner, K. H. The nucleosome map of the mammalian liver. Nat. Struct. Mol. Biol. 18, 742-746 (2011).

16. Chen, Q. et al. Metformin impairs systemic bile acid homeostasis through regulating SIRT1 protein levels. Biochim. Biophys. Acta 1864, 101-112 (2017).

17. Hoepfner, J. et al. Biphasic modulation of Wnt signaling supports efficient foregut endoderm formation from human pluripotent stem cells. Cell. Biol. Int. 40, 534-548 (2016).

18. Kitajima, K., Oki, S., Ohkawa, Y., Sumi, T. \& Meno, C. Wnt signaling regulates leftright axis formation in the node of mouse embryos. Dev. Biol. 380, 222-232 (2013).

19. Weidenfeld, J., Shu, W., Zhang, L., Millar, S. E. \& Morrisey, E. E. The WNT7b promoter is regulated by TTF-1, GATA6, and Foxa2 in lung epithelium. J. Biol. Chem. 277, 21061-21070 (2002).

20. Yeung Tsang, K., Wa Tsang, S., Chan, D. \& Cheah, K. S. The chondrocytic journey in endochondral bone growth and skeletal dysplasia. Birth. Defects Res. C Embryo. Today 102, 52-73 (2014)

21. Cohen-Kfir, E. et al. Sirt1 is a regulator of bone mass and a repressor of Sost encoding for sclerostin, a bone formation inhibitor. Endocrinology 152, 4514-4524 (2011)

22. Kandyba, E. \& Kobielak, K. Wnt7b is an important intrinsic regulator of hair follicle stem cell homeostasis and hair follicle cycling. Stem Cells 32, 886-901 (2014).

23. Wang, Z. et al. IL-17A inhibits osteogenic differentiation of bone mesenchymal stem cells via Wnt signaling pathway. Med. Sci. Monit. 23, 4095-4101 (2017).

24. Buyl, K. et al. Characterization of hepatic markers in human Wharton's Jellyderived mesenchymal stem cells. Toxicol. Vitr. 28, 113-119 (2014).

25. Loebel, C. et al. In vitro osteogenic potential of human mesenchymal stem cells is predicted by Runx2/Sox9 ratio. Tissue Eng. Part. A. 21, 115-123 (2015).

26. Ding, $Y$. et al. Overexpression of transcription factor Foxa2 and Hnflalpha induced rat bone mesenchymal stem cells into hepatocytes. Cytotechnology 68, 2037-2047 (2016).

27. Li, H. et al. FOXP1 controls mesenchymal stem cell commitment and senescence during skeletal aging. J. Clin. Invest. 127, 1241-1253 (2017).

28. Wolfrum, C., Asilmaz, E., Luca, E., Friedman, J. M. \& Stoffel, M. Foxa2 regulates lipid metabolism and ketogenesis in the liver during fasting and in diabetes. Nature 432, 1027-1032 (2004).

29. Ionescu, A. et al. FoxA family members are crucial regulators of the hypertrophic chondrocyte differentiation program. Dev. Cell. 22, $927-939$ (2012).

30. Abdallah, B. M.t Jafari, A., Zaher, W., Qiu, W. \& Kassem, M. Skeletal (stromal) stem cells: an update on intracellular signaling pathways controlling osteoblast differentiation. Bone 70, 28-36 (2015).

31. Wang, T., Zhang, X. \& Bikle, D. D. Osteogenic differentiation of periosteal cells during fracture healing. J. Cell. Physiol. 232, 913-921 (2017).

32. Gong, Y. et al. LDL receptor-related protein 5 (LRP5) affects bone accrual and eye development. Cell 107, 513-523 (2001).

33. Chen, D. et al. Differential roles for bone morphogenetic protein (BMP) receptor type $\mathrm{IB}$ and $\mathrm{IA}$ in differentiation and specification of mesenchymal precursor cells to osteoblast and adipocyte lineages. J. Cell. Biol. 142, 295-305 (1998).

34. Zhang, X., Zara, J., Siu, R. K., Ting, K. \& Soo, C. The role of NELL-1, a growth factor associated with craniosynostosis, in promoting bone regeneration. J. Dent. Res. 89, 865-878 (2010).

35. Ichida, F. et al. Reciprocal roles of MSX2 in regulation of osteoblast and adipocyte differentiation. J. Biol. Chem. 279, 34015-34022 (2004).

36. Nie, J. \& Sage, E. H. SPARC inhibits adipogenesis by its enhancement of betacatenin signaling. J. Biol. Chem. 284, 1279-1290 (2009).
37. Jin, L. et al. SFRP2 enhances the osteogenic differentiation of apical papilla stem cells by antagonizing the canonical WNT pathway. Cell. Mol. Biol. Lett. 22, 14 (2017).

38. Di Benedetto, A. et al. NURR1 downregulation favors osteoblastic differentiation of MSCs. Stem Cells Int. 2017, 7617048 (2017).

39. $\mathrm{Yu}, \mathrm{S}$. et al. Heparin-binding EGF-like growth factor and miR-1192 exert opposite effect on Runx2-induced osteogenic differentiation. Cell Death Dis. 4, e868 (2013).

40. Ashraf, S., Han, I. B., Park, H. \& Lee, S. H. Role of RHEB in regulating differentiation fate of mesenchymal stem cells for cartilage and bone regeneration. Int. J. Mol. Sci. 18, 880 (2017)

41. Wu, Y. et al. The osteogenic differentiation of PDLSCs is mediated through MEKJERK and p38 MAPK signalling under hypoxia. Arch. Oral. Biol. 58 1357-1368 (2013).

42. Ge, C., Xiao, G., Jiang, D. \& Franceschi, R. T. Critical role of the extracellular signal-regulated kinase-MAPK pathway in osteoblast differentiation and skeletal development. J. Cell. Biol. 176, 709-718 (2007).

43. Greenblatt, M. B., Shim, J. H. \& Glimcher, L. H. Mitogen-activated protein kinase pathways in osteoblasts. Annu. Rev. Cell. Dev. Biol. 29, 63-79 (2013).

44. Artigas, N., Urena, C., Rodriguez-Carballo, E., Rosa, J. L. \& Ventura, F. Mitogenactivated protein kinase (MAPK)-regulated interactions between Osterix and Runx2 are critical for the transcriptional osteogenic program. J. Biol. Chem. 289, 27105-27117 (2014).

45. Matsushita, T. et al. Extracellular signal-regulated kinase 1 (ERK1) and ERK2 play essential roles in osteoblast differentiation and in supporting osteoclastogenesis. Mol. Cell Biol. 29, 2543-5857 (2009).

46. An, S. Y. et al. Valproic acid promotes differentiation of hepatocyte-like cells from whole human umbilical cord-derived mesenchymal stem cells. Tissue Cell 46, 127-135 (2014).

47. Tsai, S. C. et al. ERK-modulated intrinsic signaling and $\mathrm{G}(2) / \mathrm{M}$ phase arrest contribute to the induction of apoptotic death by allyl isothiocyanate in MDAMB-468 human breast adenocarcinoma cells. Int. J. Oncol. 41, 2065-2072 (2012).

48. Zhang, W. et al. IGFBP7 regulates the osteogenic differentiation of bone marrow-derived mesenchymal stem cells via Wnt/beta-catenin signaling pathway. Faseb. J. 32, 2280-2291 (2018).

49. Zhang, W. et al. Overexpression of HSPA1A enhances the osteogenic differentiation of bone marrow mesenchymal stem cells via activation of the Wnt/beta-catenin signaling pathway. Sci. Rep. 6, 27622 (2016).

50. Kim, S. E. et al. 3D printed alendronate-releasing poly(caprolactone) porous scaffolds enhance osteogenic differentiation and bone formation in rat tibial defects. Biomed. Mater. 11, 055005 (2016).

51. Wei, B. et al. Effect of mesenchymal stem cells and platelet-rich plasma on the bone healing of ovariectomized rats. Stem Cells Int. 2016, 9458396 (2016).

52. Chen, E. E. M. et al. Knockdown of SIRT7 enhances the osteogenic differentiation of human bone marrow mesenchymal stem cells partly via activation of the Wnt/beta-catenin signaling pathway. Cell Death Dis. 8, e3042 (2017).

53. Mostafavinia A, Razavi S, Abdollahifar M, et al. Evaluation of the Effects of Photobiomodulation on Bone Healing in Healthy and Streptozotocin-Induced Diabetes in Rats. Photomed Laser Surg. 35, 537-545 (2017)

54. Xue, D. et al. Local delivery of HMGB1 in gelatin sponge scaffolds combined with mesenchymal stem cell sheets to accelerate fracture healing. Oncotarget 8, 42098-42115 (2017).

55. Bosemark, P., Isaksson, H. \& Tagil, M. Influence of systemic bisphosphonate treatment on mechanical properties of BMP-induced calluses in a rat fracture model: comparison of three-point bending and twisting test. J. Orthop. Res. 32, 721-726 (2014).

56. Bouxsein, M. L. et al. Guidelines for assessment of bone microstructure in rodents using micro-computed tomography. J. Bone Miner. Res. 25, 1468-1486 (2010). 